\title{
MAKE A CHOICE! VISUAL ATTENTION AND CHOICE BEHAVIOUR IN MULTIALTERNATIVE FOOD CHOICE SITUATIONS
}

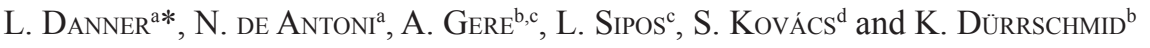

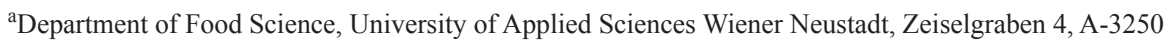 \\ Wieselburg. Austria \\ ${ }^{\mathrm{b}}$ Department of Food Science and Technology, University of Natural Resources and Life Sciences, Muthgasse 18, \\ A-1190 Vienna. Austria \\ 'Sensory Laboratory, Szent István University, H-1118 Budapest, Villányi út 29-43. Hungary \\ ${ }^{\mathrm{d}}$ Department of Research Methods and Statistics, University of Debrecen, H-4032 Debrecen, Böszörményi út 138. \\ Hungary
}

(Received: 27 January 2016; accepted: 26 April 2016)

This study investigates the relationship between gazing behaviour and choice decision in multialternative forced choice tasks, focusing on the consistency across different food product groups including apple, beer, bread, chocolate, instant soup, salad, sausage, and soft drink. Each choice task consisted of pictures of four alternatives, similar in familiarity and liking ratings, of the corresponding product group. A Tobii T60 eye-tracker was used to present the stimuli and to analyse the gazing behaviour of 59 participants during decision-making.

The results showed strong correlations between choice and gazing behaviour, in forms of more fixation counts, longer total dwell duration, and more dwell counts on the chosen alternative. No correlations for first fixation, time to first fixation, and first fixation duration were observed. These results were consistent across the eight tested product groups.

Keywords: food choice, eye-tracking, multialternative choice situation, choice prediction

Food choices are complex behaviours that are determined by many factors, including intrinsic product characteristics, biological, physiological, psychological, situational, sociocultural factors and extrinsic product characteristics and the interaction between them (KÖSTER, 2009). "The first taste is almost always with the eye" (IMrAM, 1999), triggering expectations, memories, emotions etc., consequently the visual perception is a vital component of total food quality perception and significantly influences food choice (JAROs et al., 2000; VAN DER LAAN et al., 2011).

Most published studies examined gazing behaviour in a food or nutritional context, with a special focus on package design and food labels (BIALKOVA \& VAN TRIJP, 2011; Ares et al., 2013, 2014). For a review regarding eye-tracking and nutrition label use see GrAHAM and co-workers (2012).

"Not seen, not bought" is a well-known proverb of marketing experts. This raises the question how choice in general and especially food choice, product properties, and visual attention are associated. It has been demonstrated across different tasks that several gazing parameters are correlated with choice decisions. For a recent literature review on eye movements in decision-making, see Orquin and Mueller Loose (2013). Summarizing

\footnotetext{
* To whom correspondence should be addressed.

Phone: +61 478647905; e-mail: lukas.danner@gmail.com
} 
several eye-tracking studies, they found that participants tend to have more fixations on the alternative they choose, a greater number of dwells and longer total dwell duration (dwell duration is defined as the sum of fixation durations made to a stimulus before the decision maker fixates another area). Furthermore, they stated that it is very likely that decision makers have their last fixation on the chosen alternative and likely that the first fixated alternative is the chosen one.

Although the correlation between choice and gazing behaviour has been studied for some time, only a few studies focused on food choice in particular (KRAJBich \& RANGEL, 2011; Reutskaja et al., 2011; Jantathai et al., 2013; Ares et al., 2014), and if the gazing behaviour is consistent across different food categories.

Concluding literature, there appears to be a lack of evidence showing how consistent the correlations between gazing behaviour and choice are across different food product groups. Hence, the aim of this study was to examine the correlations between gazing behaviour aspects and choice over eight different food product groups, resulting in the following hypotheses:

H1: The chosen food product receives more visual attention in form of a) more fixation counts b) longer total dwell duration and c) more dwell counts.

$\mathrm{H} 2$ : The first fixated product is more likely to be chosen than its alternatives.

H3: The last fixation before the decision is on the chosen product.

In case significant relations between choice and gazing behaviour parameters are found, it is of special interest how consistent they are, comparing the different food product groups, and how accurate these gazing parameters can predict choice, resulting in hypotheses 4.

$\mathrm{H} 4$ : The relationship between gazing behaviour and choice is consistent for different food product groups.

\section{Materials and methods}

\subsection{Visual stimuli}

Food products of everyday life, which are very familiar to the participants and have moderate visual complexity (no complex dishes), were chosen as possible stimuli. The selection of the final choice-sets and product pictures was based on a pretest with 40 participants (similar age as the participants of the main test; students aged between 18 and 28, equal gender distribution) investigating consumers familiarity, liking, and visual appearance of a wide range of products. The pretest resulted in 9-choice sets consisting of 4 pictures comparable in (high) familiarity, liking, and appealing visual appearance. Special care was taken to select pictures of good quality, comparable lightness, and size. One set out of the nine choice sets was used as a warm-up to familiarize the participants with the procedure and was not included in the data analysis. The remaining 8 choice sets represented different product categories including apple, beer, bread, chocolate, instant soup, salad, sausage, and soft drink (Fig. 1). 


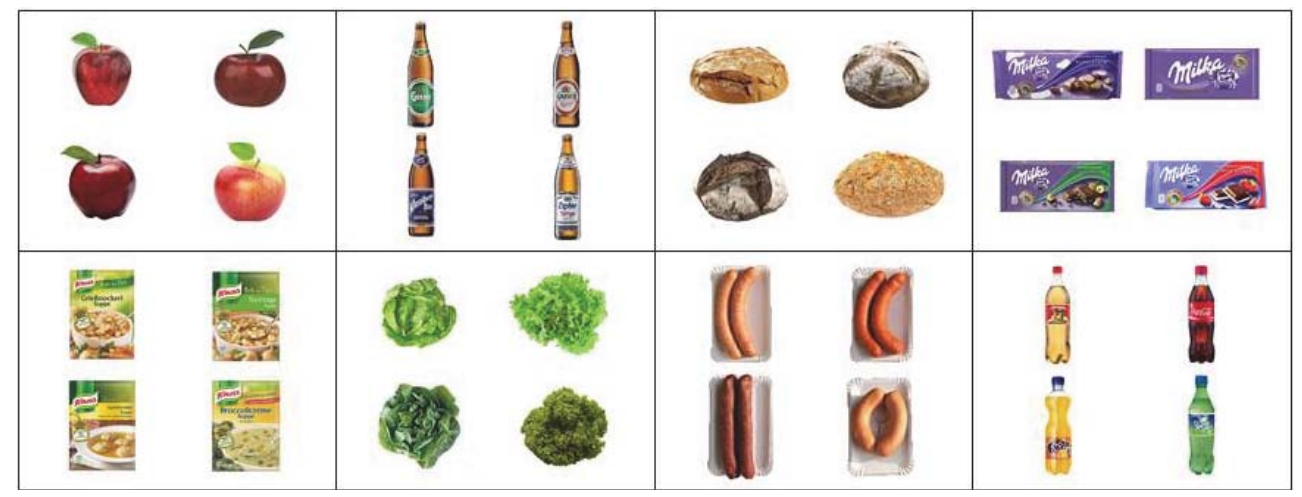

Fig. 1. The eight presented choice sets from top left to bottom right: Apple, beer, bread, chocolate, instant soup, salad, sausage, soft drink

\subsection{Eye-tracking procedure}

The same multi-alternative forced choice test paradigm (4AFC) without time limit as presented in the study by GERE and co-workers (2016) was used. Fifty-nine students of the University of Natural Resources and Life Sciences Vienna (BOKU) participated in the study (29 male, 30 female; aged between 18 and 28). A Tobii T60 eye-tracker and Tobii Studio software (version 3.0.5, Tobii Technology AB, Sweden) were used for presenting the stimuli, recording and analysing the gazing behaviour of the 59 participants during the choice task. The experiment took place under controlled environment (illumination, temperature, etc.) in the sensory laboratory of the Department of Food Science and Technology at the University of Natural Resources and Life Sciences Vienna.

Participants were instructed to look at the pictures at the monitor in a relaxed way, with the mouse in their dominant hand and not to change their sitting position during the test. After successful calibration, the test started with an instruction text on the screen explaining the procedure in detail (for a detailed flowchart of the procedure see Fig. 2). The participants were told to look at the pictures and choose the food product that appealed most to them out of the choice set, without time limitation. Before the first choice set was presented and between choice sets, a black fixation cross was displayed for $3 \mathrm{sec}$ in the centre of the white screen, to center the gazing point and standardize the starting conditions. Then the first choice set was displayed until the participants indicated their choice by clicking with the left mouse button, the mouse pointer was not visible during the decision-making. On the next screen, the mouse pointer appeared and the participants were instructed to state their choice by clicking on the chosen object. Only the data of participants stating their choice within 2 seconds after they read the instructions and directly following the mouse pointer to the object they chose (the position of the objects stayed the same as on the screen before) without investigating the other alternatives were used for the data analyses. This was necessary to ensure that the participants did not change their decision between decision-making (first click with left mouse button) and choice-stating (clicking on the product). This procedure was explained to the participants in detail before they started the test and a warm-up choice set was used to familiarize the participants with the procedure. 
Following, six eye-tracking parameters were measured: 1.) Time to first fixation (TTFF): time elapsed between the appearance of a picture and the user first fixating his/her gaze within an Area of Interest (AOI), in this case each product was defined as a separate AOI 2.) First fixation duration (FFD): time a user gazes at his/her first fixation point. 3.) Fixation duration (FD): length of a fixation (in seconds). Average FD was used for statistical analyses. 4.) Fixation count (FC): number of fixations on product. 5.) Dwell duration (DD): time elapsed between the user's first fixation on a product and the next fixation outside the product (in seconds). The total dwell duration (sum of all dwell durations on an alternative) was used during a choice task for statistical analyses. 6.) Dwell count (DC): number of "visits" to an AOI.

The gazing behaviour of the participants was recorded during the whole testing procedure, but only the gazing data during the decision-making process, starting with the presentation of the stimuli and ending when the participant stated that he or she made a decision, was used for the data analyses (stating the choice itself, by clicking on the product picture was not included).

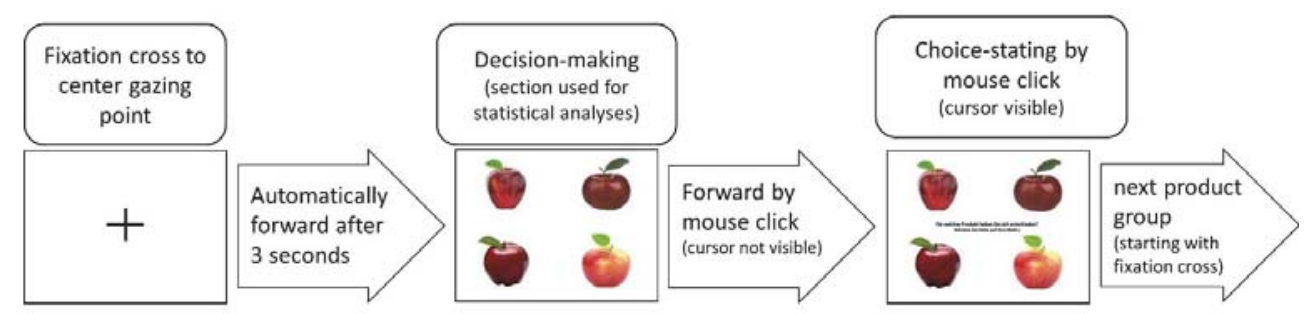

Fig. 2. Flowchart of the testing procedure on the example of apple. Eye-tracking retrieved during the decisionmaking section was used for the statistical analyses; there was no time limitation during decision-making

\subsection{Statistical analyses}

$\chi^{2}$-Square test was used to test differences in choice frequency. To investigate effects of choice and product variant on the measured gazing parameters, Repeated Measures Analysis of Variance (RMANOVA) with TTFF, FFD, FD, FC, DD, and DC as dependent measures, product variant as within subject factor, and the stated choice as between subject factor was conducted. To analyse the gazing behaviour in dependence of choice in detail, stated contrasts were used. Binomial test was used to test the relationship between first/last fixation and choice. IBM SPSS Statistics 20 (IBM Corporation, Armonk, USA) software was used to analyse the data.

\section{Results and discussion}

\subsection{Choice frequency}

Each product was chosen at least four times, and statistically significant differences in the choice frequency were found for five out of eight product sets (Fig. 3). In case of beer and salad these differences are prominent in particular; where one product alternative was chosen by less than ten percent of the participants. 


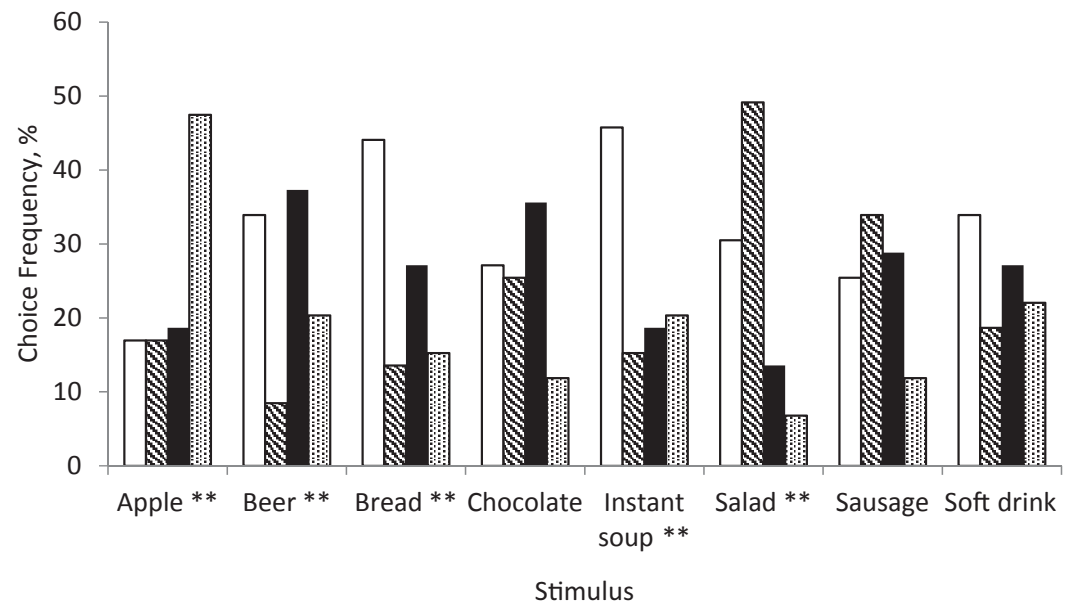

Fig. 3. Frequency of choice for all eight choice sets with each four alternatives. * indicates significant effect at a significance level of $\mathrm{P}<.05,{ }^{* *} \mathrm{P}<.01$, and $* * * \mathrm{P}<.001$. $\square$ : Product $1 ; \mathbb{\mathbb { N }}$ : Product 2; $\square$ : Product 3; 囵: Product 4

The mean decision time was the shortest for the product group soft drink with 4.7 seconds and the longest for instant soup with 6.7 seconds in average. The other six product groups ranged between these two.

\subsection{Eye-tracking Measures}

According to the results of the Repeated Measures Analysis of Variance (RMANOVA) (Table 1), the product had a significant effect on the gazing behaviour for the product groups of chocolate, salad, soda, and instant soup. No significant effect of the product choice was observed over all eight categories. However, highly significant interactions between product and choice were found for all eight product groups, indicating the chosen product was gazed at differently compared to not chosen ones.

Table 1. Results of the Repeated Measures ANOVA

\begin{tabular}{|c|c|c|c|c|c|c|c|c|c|}
\hline & & Apple & Beer & Bread & $\begin{array}{c}\text { Choco- } \\
\text { late }\end{array}$ & $\begin{array}{l}\text { Instant } \\
\text { soup }\end{array}$ & Salad & Sausage & $\begin{array}{c}\text { Soft } \\
\text { drink }\end{array}$ \\
\hline Effect & $\mathrm{df}$ & F-value & F-value & F-value & F-value & F-value & F-value & F-value & F-value \\
\hline Product & $(18,38)$ & .989 & 1.511 & .565 & $4.561 * * *$ & $2.655^{* *}$ & $3.18 * *$ & 1.356 & $2.128 * * *$ \\
\hline Choice & $(18,156)$ & .983 & 1.232 & .835 & .937 & .764 & 1.117 & .919 & 1.069 \\
\hline \multicolumn{10}{|l|}{ Product } \\
\hline $\begin{array}{l}\times \\
\text { Choice }\end{array}$ & $(54,120)$ & $3.423 * * *$ & $2.452 * * *$ & $4.345^{* * *}$ & $2.781 * * *$ & $1.803 * * *$ & $2.333 * * *$ & $2.210 * * *$ & $2.703 * * *$ \\
\hline
\end{tabular}

df: degrees of freedom

**: significant effect at a significance level of $\mathrm{P}<0.01$ and $* * * \mathrm{P}<0.001$. 


\subsection{Product effects on gazing behaviour}

Analysing the significant effects in detail, the univariate tests indicate that the product had a significant effect on TTFF for the beer category, on FD for the chocolate category, and on DD for instant soup and salad (Table 2). Furthermore, significant product effects were observed on FC and DC for salad and sausage products. Examining these effects in detail, most post hoc tests did not show significant differences.

Table 2. Results of the Repeated Measures ANOVA (RMANOVA). Factor choice is not stated due to no significant effects observed in RMANOVA

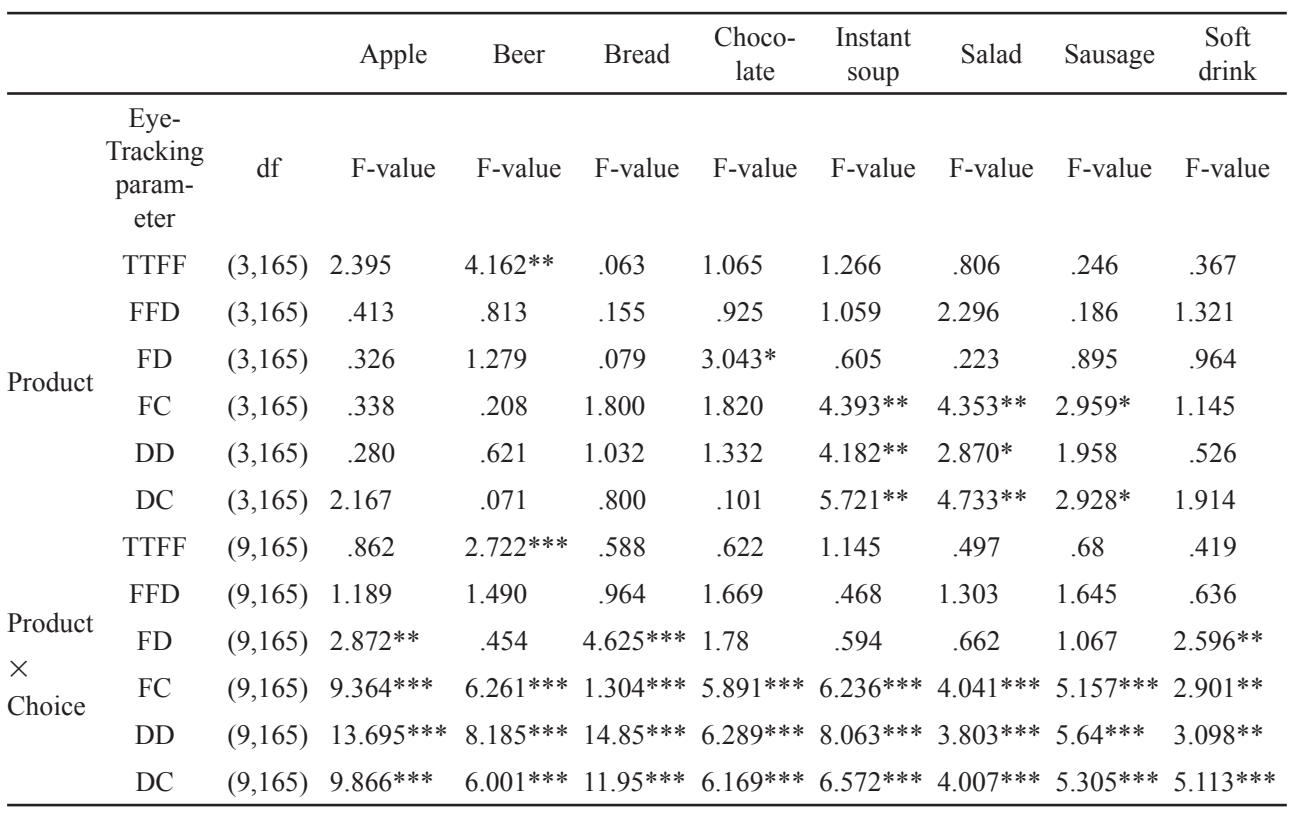

df: degrees of freedom

*: significant effect at a significance level of $\mathrm{P}<0.05$, ** $\mathrm{P}<0.01$, and *** $\mathrm{P}<0.001$.

H1: The chosen food product receives more visual attention

Significant interactions between product and choice were observed for several eyetracking parameters including TTFF, FD, FC, DD, and DC (Table 2). The latter three were highly significant for all eight product categories. These results indicate that there are clear differences between the visual attention for a chosen product and the non-chosen alternatives.

H1a: The chosen food product receives more visual attention in form of more fixation counts

Investigating fixation counts for all eight choice sets, in dependence of the stated choice, the chosen product variant received in 31 out of 32 cases more fixations than each of the not chosen alternatives, confirming hypothesis H1a (Table 3). In 17 cases, the within subject contrasts showed that the chosen object received significantly more fixations than the three other alternatives. duration

$\mathrm{H} 1 \mathrm{~b}$ : The chosen food product receives more visual attention in form of longer dwell 
Results for dwell duration are very similar to fixation counts. Dwell duration was in 31 out of 32 cases longer for the chosen product, then for each of the corresponding alternatives, which confirms hypothesis H1b. These differences were significant in 24 cases.

H1c: The chosen food product receives more visual attention in form of more dwell counts

The chosen product received in 30 out of 32 cases more dwell counts than each of the alternatives, confirming hypothesis H1c. In 20 cases the within subject contrasts showed that the chosen object received significantly more dwells than the three other alternatives.

Summarizing H1, the chosen food products received more visual attention in forms of a) more fixation counts, b) longer total dwell duration, and c) more dwell counts. This supports our hypothesis for all three parameters and is in accordance with the findings of Orquin and Mueller Loose (2013) and Jantathai and co-workers (2013).

$\mathrm{H} 2$ : The first fixated product is more likely to be chosen than its alternatives

Using the Binominal test no indication was found that the product first fixated was chosen more often than the other products, $(\mathrm{P}>0.05)$ across all eight product groups (Fig. 4A). Therefore, $\mathrm{H} 2$ could not be confirmed with this study and supports the findings of vaN DER LAAN and co-workers (2015).

H3: The last fixation before the decision is on the chosen alternative

The last fixated alternative was chosen between $88 \%$ in case of the bread choice set and $69 \%$ for the salad choice set (Fig. 4B). The Binominal test showed that the last fixated alternative was significantly more often chosen then expected by chance $(\mathrm{P}<0.001)$ for all eight choice sets, therefore supporting hypothesis $\mathrm{H} 3$ as well the findings of ORQUiN and MuelLer LoOse (2013).
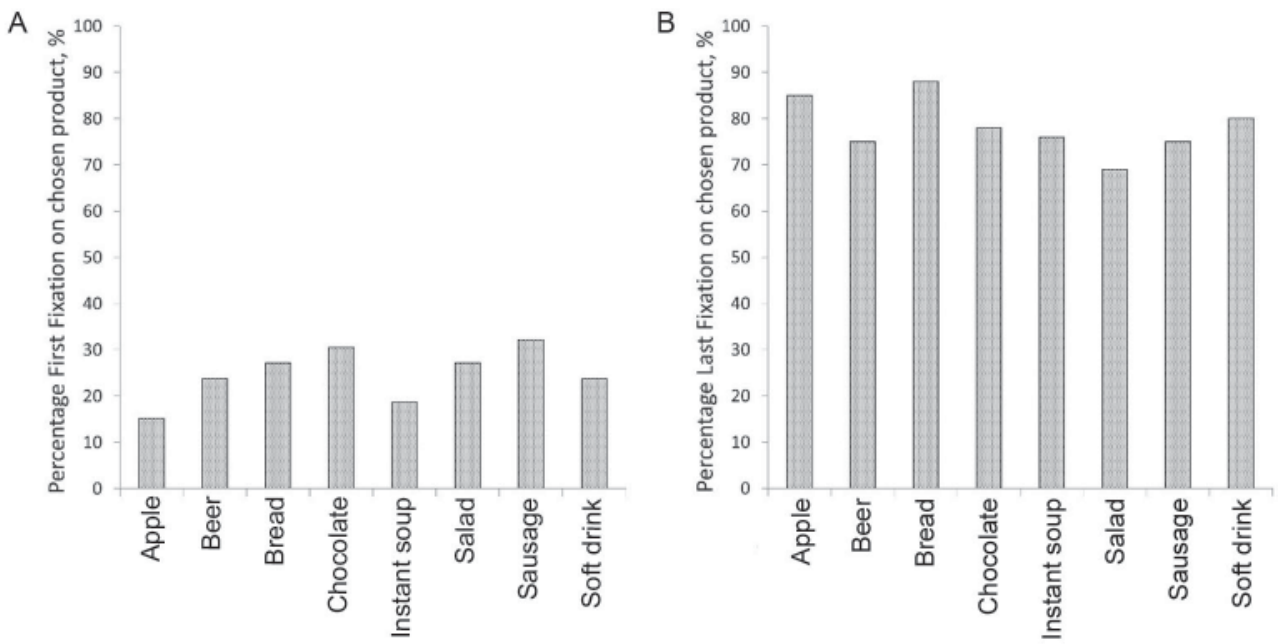

Fig. 4. Relationship between A: First fixation and B: Last fixation and product choice 


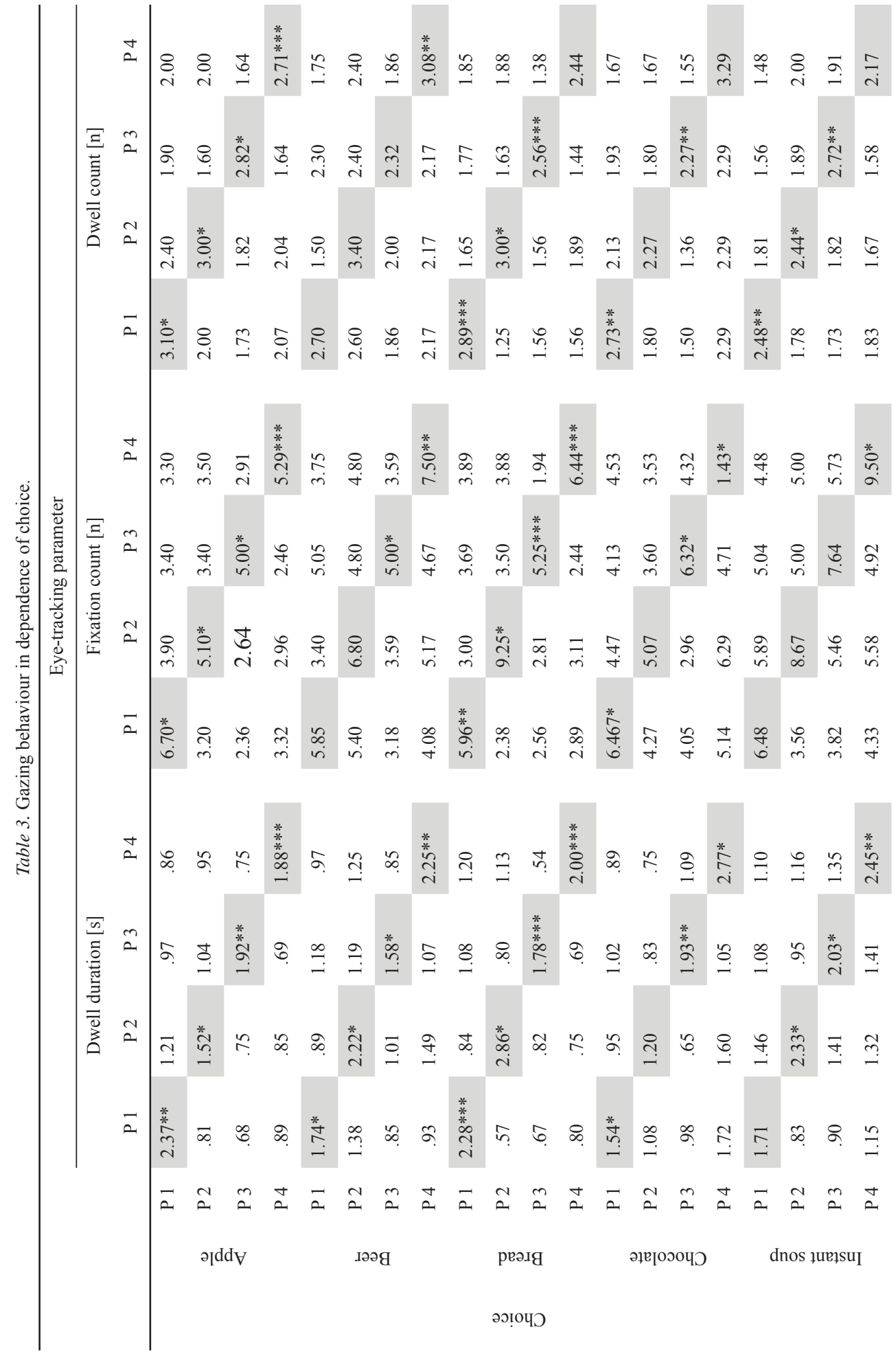




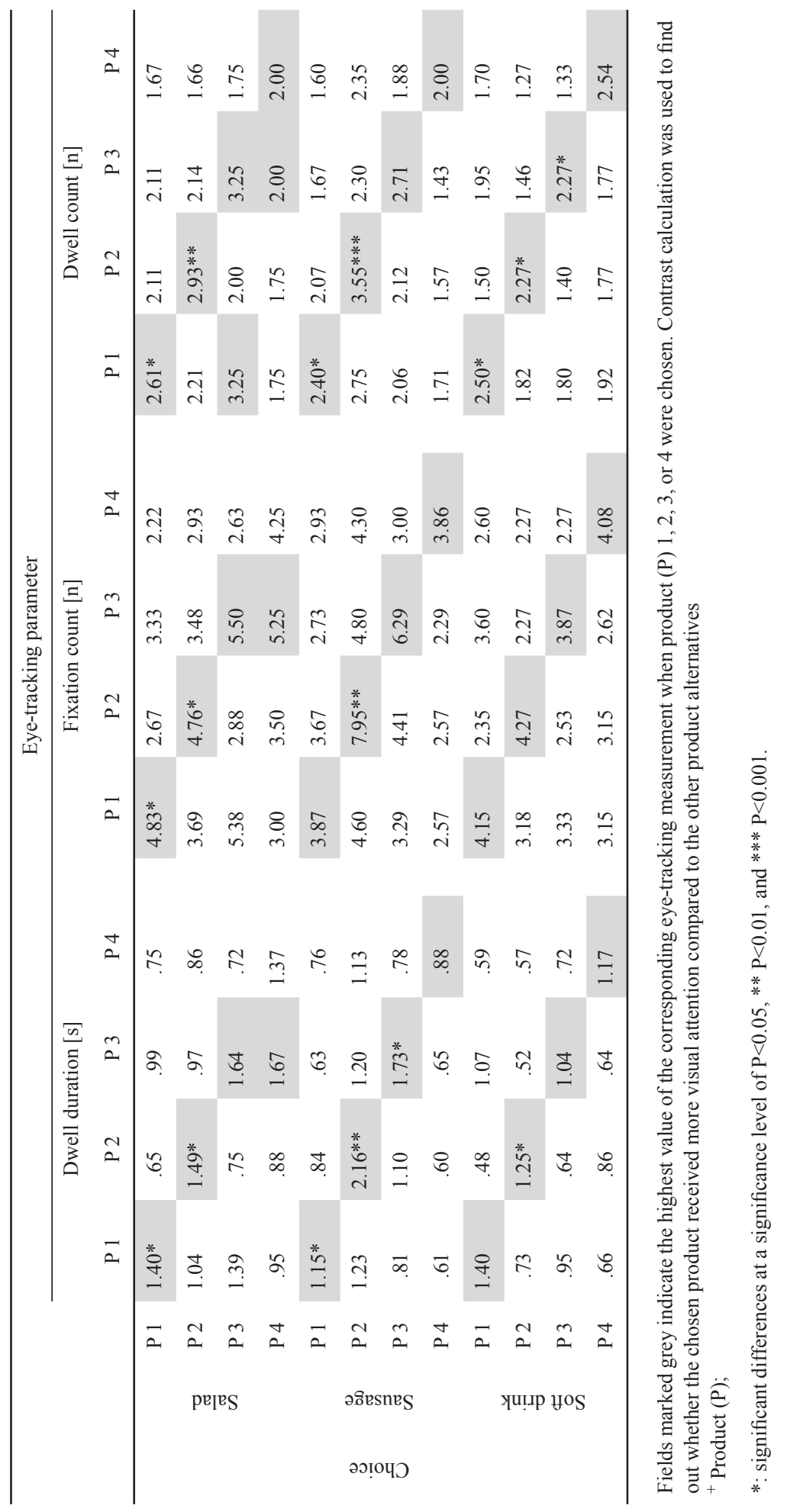


H4: The relationship between gazing behaviour and choice is consistent for different food product groups

Summarizing the results of the RMANOVA and the subsequent tests (Tables 2 and 3, Fig. 4), the relationships between choice and gazing behaviour are very consistent across the eight choice sets supporting H4.

It must be remarked that the presented study was conducted using exclusively university students aged between 18 and 28 as subjects. Therefore, generalization of the results for the general population is not possible.

\section{Conclusions}

By examining the gazing behaviour during multialternative choice tasks using eye-tracking technology, very strong correlations between choice and gazing behaviour, in forms of more fixation counts, longer total dwell duration, and more dwell counts, on the chosen alternative were found. These results were consistent across all eight tested product groups.

\section{References}

Ares, G., Giménez, A., Bruzzone, F., Vidal, L., Antúnez, L. \& Maiche, A. (2013): Consumer visual processing of food labels: Results from an eye-tracking study. J. Sens. Stud., 28(2), 138-153.

Ares, G., Mawad, F., Giménez, A. \& Maiche, A. (2014): Influence of rational and intuitive thinking styles on food choice: Preliminary evidence from an eye-tracking study with yogurt labels. Food Qual. Prefer., 31, 28-37.

Bialkova, S. \& van TriJP, H.C.M. (2011): An efficient methodology for assessing attention to and effect of nutrition information displayed front-of-pack. Food Qual. Prefer., 22, 592-601.

Gere, A., Danner, L., de Antoni, N., Kovács, S., Dürrschmid, K. \& Sipos, L. (2016): Visual attention accompanying food decision process: an alternative approach to choose the best models. Food Qual. Prefer., 51, 1-7.

Graham, D.J., Orquin, J.L. \& Visschers, V.H.M. (2012): Eye tracking and nutrition label use: A review of the literature and recommendations for label enhancement. Food Policy, 37, 378-382.

Imram, N. (1999): The role of visual cues in consumer perception and acceptance of a food product. Nutr. Food Sci., 99(5), 224-230.

Jantathai, S., Danner, L., Joechl, M. \& Dürrschmid, K. (2013): Gazing behavior, choice and color of food: Does gazing behavior predict choice? Food Res. Int., 54(2), 1621-1626.

Jaros, D., Rohm, H. \& Strobl, M. (2000): Appearance properties - A significant contribution to sensory food quality? LWT - Food Sci. Technol., 33, 320-326.

KösTER, E.P. (2009): Diversity in the determinants of food choice: A psychological perspective. Food Qual. Prefer., 20(2), 70-82.

KrajBich, I. \& Rangel, A. (2011): Multialternative drift-diffusion model predicts the relationship between visual fixations and choice in value-based decisions. P. Natl. Acad. Sci. (PNAS), 108(33), 13852-13857.

Orquin, J.L. \& Mueller Loose, S. (2013): Attention and choice: A review on eye movements in decision making. Acta Psychol., 144(1), 190-206.

Reutskaja, E., Nagel, R., Camerer, C. \& Rangel, A. (2011): Search dynamics in consumer choice under time pressure: An eye-tracking study. Am. Econ. Rev., 101(2), 900-926

VAn der LaAn, L.N., De Ridder, D.T.D., Viergever, M.A. \& Smeets, P A.M. (2011): The first taste is always with the eyes: a meta-analysis on the neural correlates of processing visual food cues. NeuroImage, 55(1), $296-303$.

van der Laan, L.N., Hooge, I.T.C., de Ridder, D.T.D., Viergever, M.A. \& Smeets, P.A.M. (2015): Do you like what you see? The role of first fixation and total fixation duration in consumer choice. Food Qual. Prefer., 39, 4655. 